\title{
Minor-monotone crossing number
}

\author{
Drago Bokal ${ }^{1}$, Gašper Fijavž ${ }^{\dagger}$ and Bojan Mohar ${ }^{3 \ddagger}$ \\ ${ }^{1}$ Institute for Mathematics, Physics, and Mechanics, Jadranska 19, 1000 Ljubljana, Slovenia \\ ${ }^{2}$ Faculty of Computer and Information Science, University of Ljubljana, Tržaška 25, 1000 Ljubljana, Slovenia \\ ${ }^{3}$ Department of Mathematics, University of Ljubljana, Jadranska 19, 1000 Ljubljana, Slovenia
}

The minor crossing number of a graph $G, \operatorname{mcr}(G)$, is defined as the minimum crossing number of all graphs that contain $G$ as a minor. We present some basic properties of this new minor-monotone graph invariant. We give estimates on mcr for some important graph families using the topological structure of graphs satisfying $\operatorname{mcr}(G) \leq k$.

Keywords: crossing number, graph minor, minor-monotone graph parameter

\section{Contents}

1 Introduction $\quad 123$

2 Definitions 124

3 Origins of the minor crossing number $\quad 124$

4 Complete graphs $\quad 125$

5 Other graphs, other results $\quad 126$

\section{Introduction}

Crossing number is an intriguing graph parameter. Once we take care of the technical topological prerequisites (which we do not mention to the general public in the first place) drawing a graph with as few crossings as possible is a task with appeal even to non-specialists. Even though the bibliography on crossing number is extensive [11], few exact results on the crossing number are known and new ideas seem to be needed.

Crossing numbers of graphs are beleived to have application in VLSI design. Chip manufacturers devise huge electrical networks with as few crossing wires as possible $[1,5,6]$. However, vertices of large

\footnotetext{
${ }^{\dagger}$ corresponding author, email:gasper.fijavz@fri.uni-lj.si

$¥$ All authors supported in part by the Ministry of Higher Education, Science, and Technology of Slovenia, Research Program L1-5014-0101-04.

1365-8050 (c) 2005 Discrete Mathematics and Theoretical Computer Science (DMTCS), Nancy, France
} 
degree are often replaced by binary trees, which makes the notion of minor crossing number more natural than the traditional crossing number in VLSI design.

Crossing number of a graph $G$ measures its nonplanarity, which seems to be related to the global structure of $G$. Unfortunately, crossing number does not collaborate well with either graph minors or embedding properties of graphs, the two most important structural graph concepts.

We suggest a possible approach to this problem, how to relate crossing number to graph minors and graph embeddings. The motivation comes form the definition of singly crossing graphs from [9] and a construction of minor-monotone graph invariants from [3]. Finally let us note that results in this abstract were taken from [2] and will be published in greater detail elsewhere.

\section{Definitions}

Let $G=(V, E)$ be a graph and let $S_{0}$ denote the 2-sphere. We shall tacitly assume that all surfaces are triangulated [8, Theorem 3.1.1] and denote the closed surface of nonorientable genus $k$ by $\mathbb{N}_{k}$. Further we assume the folklore definitions of PL nice drawings of graphs. Let $D$ be a drawing of a graph $G$ in $S_{0}$. The crossing number of $D, \operatorname{cr}(D)$, is the number of crossings of $D$, and the crossing number of a graph $G, \operatorname{cr}(G)$, is the minimal $\operatorname{cr}(D)$ taken over all possible drawings of $G$.

For a given graph $G$, the minor crossing number is defined as the minimum crossing number of all graphs, which contain $G$ as a minor:

$$
\operatorname{mcr}(G):=\min \{\operatorname{cr}(H) \mid G \text { is a minor of } H\} .
$$

It is easy to see that mcr is minor-monotone: if $H$ is a minor of $G$ then $\operatorname{mcr}(H) \leq \operatorname{mcr}(G)$. Similar approach can be applied to every graph parameter and the resulting graph parameter is always minormonotone.

Let $G$ be a graph with $\operatorname{mcr}(G)=k$. If $G$ is a minor of $\hat{G}$ and $\operatorname{cr}(\hat{G})=k$ we say that $\hat{G}$ is a realizing graph of $G$, and a drawing $\hat{D}$ of $\hat{G}$ with exactly $k$ crossings is a realizing drawing of $G$.

An alternative view of the minor crossing number of $G$ is the following. Replace each vertex $v \in V(G)$ with a vertex tree $T_{v}$ and make sure that trees $T_{v}$ and $T_{w}$ are connected with an edge if $u v \in E(G)$, and let us call the newly obtained graph $G^{\prime}$. Let $D^{\prime}$ be the drawing of $G^{\prime}$. Now the crossings of $D^{\prime}$ come in three different flavors: vertex-vertex crossings if an edge of $T_{v}$ crosses an edge of $T_{u}$, vertex-edge crossings if an edge of a tree $T_{v}$ crosses an edge which connects two vertex trees, and edge-edge crossings if two edges connecting pairs of vertex trees cross. Optimizing over all possible choices for vertex trees and their connections and over all possible drawings of these graphs yields a realizing drawing of $G$.

\section{Origins of the minor crossing number}

Minor-monotone graph parameter such as mcr blends well with the theory of graph-minors. Given $k$, the class of graphs satisfying $\operatorname{mcr}(G) \leq k$ is minor closed, and can be characterized by excluding a finite set of forbidden minors.

Almost by definition $\operatorname{cr}(G) \leq 0$ if and only if $G$ is planar, and it is easy to see that also $\operatorname{mcr}(G) \leq 0$ in this case.

The next step was settled by Robertson and Seymour [9].

Theorem 3.1 (Robertson and Seymour) For every graph $G$ the following are equivalent: 

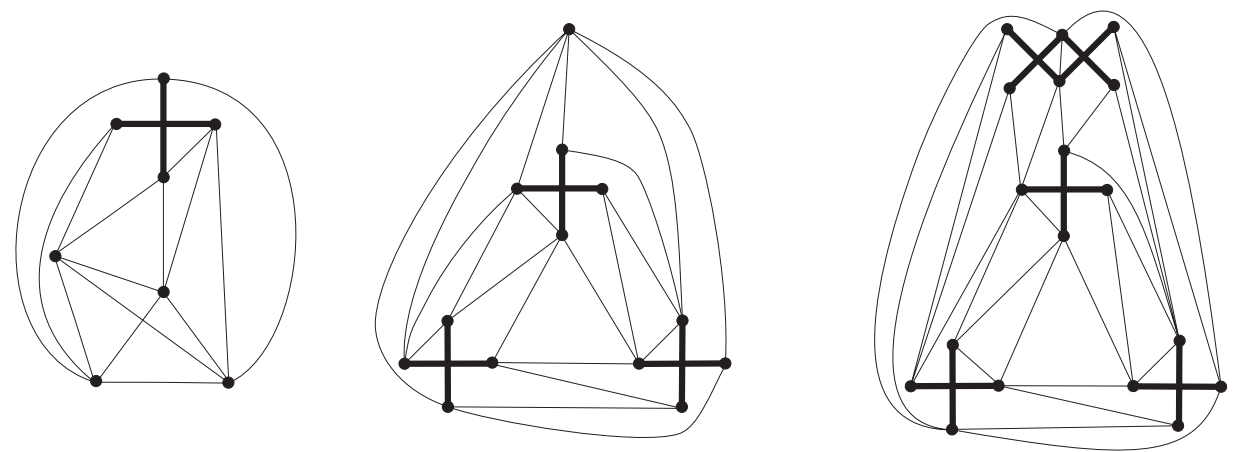

Fig. 1: Realizing drawings of $K_{6}, K_{7}$, and $K_{8}$, respectively, where thick edges represent vertex trees.

1. $\operatorname{mcr}(G) \leq 1$.

2. G admits an embedding in the projective-plane with face width at most 2.

3. $G$ does not contain a minor from $\left\{G_{1}, \ldots, G_{35}, Q_{1}, \ldots, Q_{6}\right\}$ where $G_{1}, \ldots, G_{35}$ are forbidden minors for embeddability in the projective plane and $Q_{1}, \ldots, Q_{6}$ are projective planar graphs that can be obtained from the Petersen graph by successively applying the $Y \Delta$ and $\Delta Y$ operations.

\section{Complete graphs}

In this section we shall invite the reader on a journey of estimating the minor crossing number of complete graphs. Similar reasoning can be applied to other graphs as well.

As the Figure 1 shows $\operatorname{mcr}\left(K_{6}\right)=1, \operatorname{mcr}\left(K_{7}\right)=3$, and $\operatorname{mcr}\left(K_{8}\right)=5$. To be precise, these drawings serve merely as upper bounds on the minor crossing number. Observe that all crossings shown are vertexvertex crossings.

Let us argue that $\operatorname{mcr}\left(K_{8}\right)=5$. Let $G^{\prime}$ be a realizing graph of $K_{8}$. Assume first that $\operatorname{mcr}\left(K_{8}\right)=$ $\operatorname{cr}\left(G^{\prime}\right) \leq 3$. Let $D^{\prime}$ be a drawing of $G^{\prime}$ with three crossings. In this case we can paste a crosscap in the close neighborhood of every crossing of $D^{\prime}$ and obtain an embedding of $G^{\prime}$ in $\mathbb{N}_{3}$. By contracting appropriately we obtain an embedding of $K_{8}$ in $\mathbb{N}_{3}$ which is absurd, as the nonorientable genus of $K_{n}$ is equal to $\lceil(n-3)(n-4) / 6\rceil[8]$.

It could still be possible that $\operatorname{mcr}\left(K_{8}\right)=4$. Following the above trick we obtain an embedding of $K_{8}$ in $\mathbb{N}_{4}$ with a special topological structure. Such an embedding admits a collection of 4 pairwise noncrossing onesided curves which intersect the embedding of $K_{8}$ in at most 2 vertices each. Using homology group of $\mathbb{N}_{4}$ we show [2] that we can add another $4-1$ edges to the embedding of $K_{8}$ in order to obtain an embedding of a graph with 8 vertices, 31 edges (obviously some of them are parallel), but without creating faces of length two. Using Euler formula we then prove the following result [2].

Theorem 4.1 Let $G$ be a simple graph with $n=|V(G)|$ and $m=|E(G)|$. Then

$$
\operatorname{mcr}(G) \geq \frac{1}{2}(m-3 n+6)
$$


An application of Theorem 4.1 on complete graphs yields the following lower bound inequality, which is sharp for $3 \leq n \leq 8$.

Proposition 4.2 Let $n \geq 3$. Then $\operatorname{mcr}\left(K_{n}\right) \geq\left\lceil\frac{1}{4}(n-3)(n-4)\right\rceil$.

The appropriate upper bound inequlity is obtained using examples. Choose an integer $n$. Let $V_{a}$ be the graph of the function $|x-a|$ for values $x \in[-n+a, n+a]$. Choosing $V_{0}, V_{1}, \ldots, V_{n}$ as vertex trees and connecting pairs of vertex trees close to where they cross we obtain a drawing of a graph $G$, which contains $K_{n}$ as a minor. This shows that $\operatorname{mcr}\left(K_{n}\right) \leq(n-1) n / 2$. By carefully drawing some additional vertex trees close to the drawing described above we obtain the following upper bound:

Proposition 4.3 Let $n \geq 9$. Then $\operatorname{mcr}\left(K_{n}\right) \leq\left\lfloor\frac{1}{2}(n-5)^{2}\right\rfloor+3$.

\section{Other graphs, other results}

Using similar techniques as in the case of complete graphs we obtain the following bounds for minor crossing numbers of complete bipartite graphs.

Proposition 5.1 Let $n \geq m \geq 3$. Then

$$
\left\lfloor\frac{1}{2}(m-2)(n-2)\right\rfloor \leq \operatorname{mcr}\left(K_{m, n}\right) \leq(m-3)(n-3)+5
$$

Using the best currently known bounds on the crossing number of hypercubes by Sýkora, Vrto [10] and Madej [7] we can estimate their minor crossing numbers:

Proposition 5.2 Let $n \geq 4$. Then

$$
\frac{1}{n^{2}}\left(\frac{1}{5} 4^{n}-2^{n+1}\right)-2^{n+1}<\operatorname{mcr}\left(Q_{n}\right) \leq 2^{n-2}\left(7 \cdot 2^{n-5}+2 n-4\right) .
$$

Moreno and Salazar [4] have proven the following theorem:

Theorem 5.3 (Moreno and Salazar) Let $G$ be a minor of a graph $H$ with $\Delta(G) \leq 4$. Then

$$
\frac{1}{4} \operatorname{cr}(G) \leq \operatorname{cr}(H)
$$

Using weighted graphs and counting crossings according to edge weight we have obtained the following generalization of Theorem 5.3, which is particularly useful at estimating the minor crossing number of sparse graphs.

Theorem 5.4 Let $G$ be a minor of a graph $H$ and $\tau:=\left\lfloor\frac{1}{2} \Delta(G)\right\rfloor$. Then

$$
\operatorname{cr}(G) \leq \tau^{2} \operatorname{cr}(H)
$$

This implies that we can estimate the minor crossing number with the use of crossing number and maximum degree of a graph.

Corollary 5.5 Let $G$ be a graph and $\tau:=\left\lfloor\frac{1}{2} \Delta(G)\right\rfloor$. Then

$$
\operatorname{mcr}(G) \leq \operatorname{cr}(G) \leq \tau^{2} \operatorname{mcr}(G) .
$$


We have to note that in the case of complete graphs embedding properties of comlpete graphs yield better lower bound than an application of Corollary 5.5.

We were able to show that minor crossing number is block-additive.

Proposition 5.6 If $G_{1}, \ldots, G_{b}$ are blocks of a graph $G$, then

$$
\operatorname{mcr}(G)=\operatorname{mcr}\left(G_{1}\right)+\ldots+\operatorname{mcr}\left(G_{b}\right) .
$$

On the other hand, when trying to reduce the computation of $\operatorname{mcr}(G)$ to the 3-connected components of $G$ we have run into subtle difficulties. So let us conclude with the question:

Question 5.7 Let $\left(G_{1}, G_{2}\right)$ be a 2-separation of $G$, and assume that the corresponding separator vertices are $u$ and $v$. How does $\operatorname{mcr}(G)$ relate to $\operatorname{mcr}\left(G_{1}+u v\right)$ and $\operatorname{mcr}\left(G_{2}+u v\right)$ ? Is it true that $\operatorname{mcr}(G)=$ $\operatorname{mcr}\left(G_{1}+u v\right)+\operatorname{mcr}\left(G_{2}+u v\right) ?$

\section{References}

[1] S. N. Bhatt and F. T. Leighton. A framework for solving VLSI graph layout problems. J. Comput. System Sci., 28(2):300-343, 1984.

[2] D. Bokal, G. Fijavž, and B. Mohar. The minor crossing number. submitted, 2005.

[3] G. Fijavž. Graph minors and connectivity (in Slovene). PhD thesis, University of Ljubljana, Slovenia, 2001.

[4] E. Garcia-Moreno and G. Salazar. Bounding the crossing number of a graph in terms of the crossing number of a minor with small maximum degree. J. Graph Theory, 36(3):168-173, 2001.

[5] F. T. Leighton. Complexity issues in VLSI. MIT Press, Cambridge, MA, 1983.

[6] F. T. Leighton. New lower bound techniques for VLSI. Math. Systems Theory, 17(1):47-70, 1984.

[7] T. Madej. Bounds for the crossing number of the $N$-cube. J. Graph Theory, 15(1):81-97, 1991.

[8] B. Mohar and C. Thomassen. Graphs on surfaces. Johns Hopkins Studies in the Mathematical Sciences. Johns Hopkins University Press, Baltimore, MD, 2001.

[9] N. Robertson and P. Seymour. Excluding a graph with one crossing. In Graph structure theory (Seattle, WA, 1991), volume 147 of Contemp. Math., pages 669-675. Amer. Math. Soc., Providence, RI, 1993.

[10] O. Sýkora and I. Vřto. On crossing numbers of hypercubes and cube connected cycles. BIT, 33(2):232-237, 1993.

[11] I. Vřto. Crossing number of graphs: A bibliography. ftp://ftp.ifi.savba.sk/pub/imrich/crobib.pdf. 
